\title{
Electrospun Rare-Earth Orthovanadate Scintillator Nanofibers Analyzed by EPMA
}

\author{
Jessa Guffie, Carlos Posada, Gibin George, Shubo Han and Zhiping Luo* \\ Department of Chemistry and Physics, Fayetteville State University, Fayetteville, NC USA \\ *Corresponding author: zluo@uncfsu.edu
}

Luminescent rare-earth $(\mathrm{R})$ orthovanadates $\left(\mathrm{RVO}_{4}\right)$ fall into the mineral group wakefieldite, crystallizing in zircon type $\left(\mathrm{I}_{1} /\right.$ amd $)$ structure which consists of $\mathrm{VO}_{4}$ tetrahedra sharing corners and edges with $\mathrm{RO}_{8}$ dodecahedra [1]. The rare-earth orthovanadates are recognized widely as optical maser materials. Moreover, they are identified as potential candidates for optical sensing [2], photocatalytic degradation of textile effluents [3], hosts for luminescent ions [4], battery electrodes [5], etc. The plausible applications of several orthovanadates are not addressed very well in the literature. The hightemperature stability and the relatively high concentration of rare-earth ions in the rare-earth orthovanadates make them ideal candidates for scintillator materials [6-8]. Large $\mathrm{RVO}_{4}$ crystals are commercially used as a promising candidate for magneto-optical applications in visible and nearinfrared regions [9]; however, the nanosized counterparts of $\mathrm{RVO}_{4}$ are multifunctional with magnetic, photocatalytic and antibacterial properties [10]. In this work, we fabricated nanosized orthovanadate fibers using electrospinning method and confirmed the morphology and composition using an Electron Probe Microanalyzer (EPMA).

0.07 $\mathrm{M}$ ammonium metavanadate and 0.07 $\mathrm{M}$ terbium (III) nitrate were dissolved in a $20 \mathrm{~mL} \mathrm{20/40/40}$ mixture of $\mathrm{H}_{2} \mathrm{O}_{2} /$ Ethanol/ $N, N$-Dimethylformamide to prepare the electrospinnable precursor solution. Then $3.0 \mathrm{~g}$ of polyvinylpyrrolidone (PVP) (molecular weight $\sim 1,200,000 \mathrm{~g} / \mathrm{mol}$ ) was added to the above solution and stirred vigorously for $12 \mathrm{~h}$ to ensure uniform mixing. The electrospinning was conducted at atmospheric conditions with an applied voltage of $18 \mathrm{kV}$, a flow rate of $300 \mu \mathrm{L} / \mathrm{h}$, and a spinneret to collector distance of $17 \mathrm{~cm}$. The xerogel fibers obtained through electrospinning process were subsequently calcined at $700{ }^{\circ} \mathrm{C}$ in air for $5 \mathrm{~h}$ at a ramp of $1{ }^{\circ} \mathrm{C} / \mathrm{min}$ to obtain the orthovanadate nanofibers. Samples were coated with carbon and analyzed in a JEOL field-emission JXA-8530F EPMA, which was equipped with an SDD X-ray energy-dispersive spectrometer (EDS), and five wavelength-dispersive spectrometers (WDSs) worked at $15 \mathrm{kV}$.

The SEM images of $\mathrm{RVO}_{4}$ nanofibers after calcination are shown in Fig. 1 (a, b). The observed size of the $\mathrm{RVO}_{4}$ fibers are $<150 \mathrm{~nm}$. The diameter of the fibers is significantly reduced upon calcination as compared to xerogel precursor fibers. The EDS analysis, as shown in Fig. 1(c), confirms that the composition of the obtained fibers has the significant peaks arising from $\mathrm{Tb}, \mathrm{V}$ and $\mathrm{O}$. The composition is also validated with WDS analysis, which clearly reveals the stoichiometric composition to that of anticipated $\mathrm{RVO}_{4}[11]$.

References:

[1] G. Picardi, et. al., Electrochim. Acta. 44 (1999), p. 3157.

[2] N. Duée, et. al., Chem. Mater. 27 (2015), p, 5198.

[3] V. Sivakumar, et. al., J. Mater. Sci. Mater. Electron. 28 (2017), p. 4014. 
[4] Y. Ding, et. al., J. Korean Ceram. Soc. 54 (2017), p. 96.

[5] F. Artuso, et. al., Electrochim. Acta. 46 (2001), p. 2077.

[6] Z. Luo, et al., Curr. Nanosci. 13 (2017), p. 364.

[7] G. George, et al., J. Mater. Chem. C 6 (2018), p. 7285.

[8] G. George, et al., RSC Adv. 8 (2018), p. 39296.

[9] Y. Zhu, et. al., Opt. Mater. 65 (2017), p. 106.

[10] F. Ahmadi, et. al., J. Mater. Sci. Mater. Electron. 28 (2017), p. 14362.

[11] This work was supported by NSF PREM program DMR 1827731, and DoD ARO W911NF1810469.
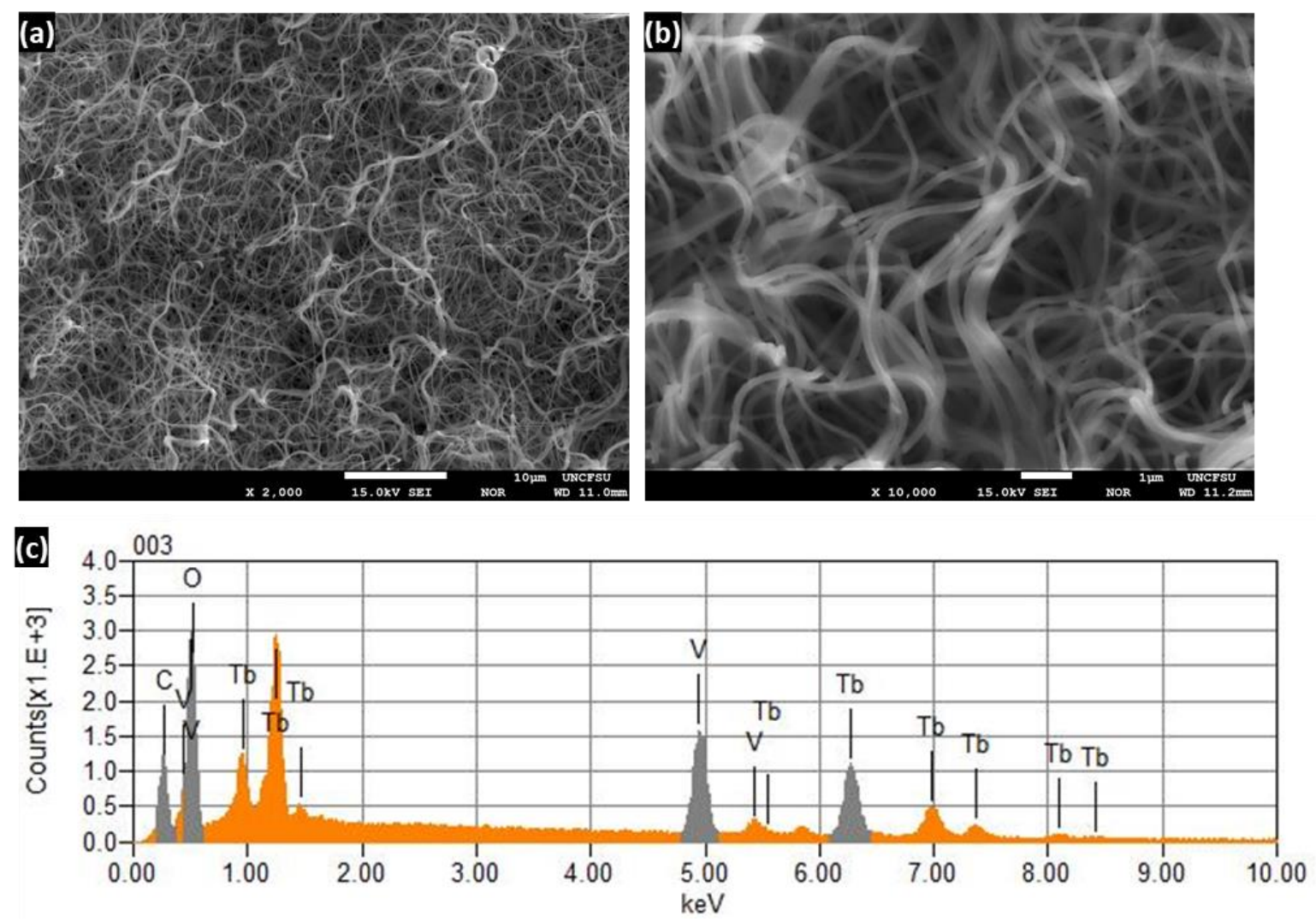

Figure 1. (a) Low and (b) high magnification SEM images of electrospun $\mathrm{RVO}_{4}$ scintillator nanofibers, and (c) the corresponding EDS spectrum.

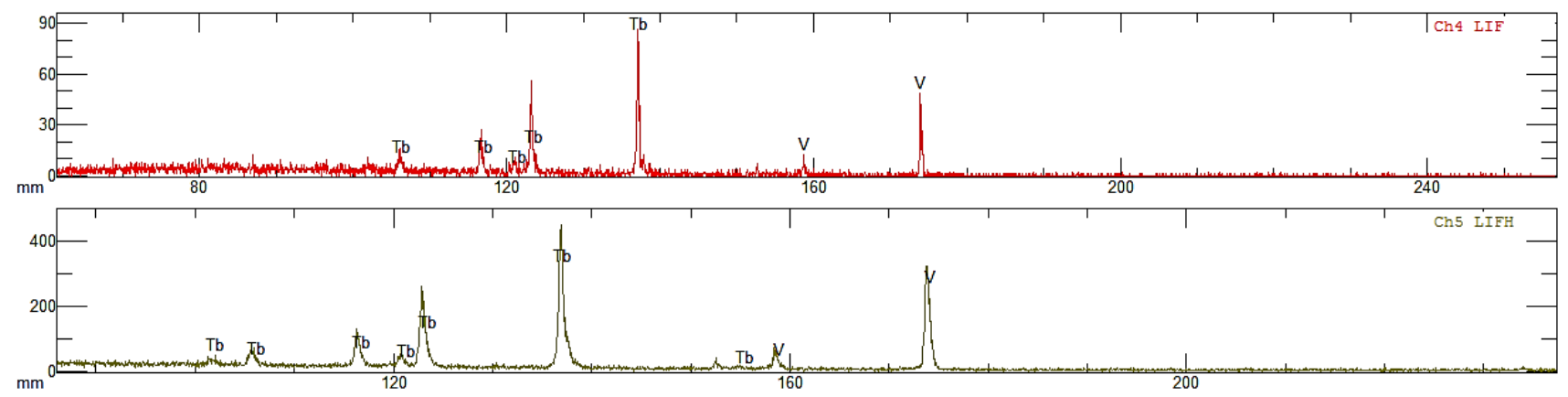

Figure 2. WDS spectra of $\mathrm{RVO}_{4}$ nanofibers obtained using different crystals. 